\title{
Inhibition of cell proliferation does not slow down echinoderm neural regeneration
}

\author{
Vladimir S. Mashanov ${ }^{1,2^{*}}$ (D) $^{\dagger}$, Olga R. Zueva ${ }^{1,2 \dagger}$ and José E. García-Arrarás²
}

\begin{abstract}
Background: Regeneration of the damaged central nervous system is one of the most interesting post-embryonic developmental phenomena. Two distinct cellular events have been implicated in supplying regenerative neurogenesis with cellular material - generation of new cells through cell proliferation and recruitment of already existing cells through cell migration. The relative contribution and importance of these two mechanisms is often unknown.

Methods: Here, we use the regenerating radial nerve cord (RNC) of the echinoderm Holothuria glaberrima as a model of extensive post-traumatic neurogenesis in the deuterostome central nervous system. To uncouple the effects of cell proliferation from those of cell migration, we treated regenerating animals with aphidicolin, a specific inhibitor of S-phase DNA replication. To monitor the effect of aphidicolin on DNA synthesis, we used BrdU immunocytochemistry. The specific radial glial marker ERG1 was used to label the regenerating RNC. Cell migration was tracked with vital staining with the lipophilic dye Dil.

Results: Aphidicolin treatment resulted in a significant 2.1-fold decrease in cell proliferation. In spite of this, the regenerating RNC in the treated animals did not differ in histological architecture, size and cell number from its counterpart in the control vehicle-treated animals. Dil labeling showed extensive cell migration in the RNC. Some cells migrated from as far as $2 \mathrm{~mm}$ away from the injury plane to contribute to the neural outgrowth.

Conclusions: We suggest that inhibition of cell division in the regenerating RNC of $\mathrm{H}$. glaberrima is compensated for by recruitment of cells, which migrate into the RNC outgrowth from deeper regions of the neuroepithelium. Neural regeneration in echinoderms is thus a highly regulative developmental phenomenon, in which the size of the cell pool can be controlled either by cell proliferation or cell migration, and the latter can neutralize perturbations in the former.
\end{abstract}

Keywords: Echinoderm, Central nervous system, Regeneration, Neurogenesis, Cell migration, Cell division

\section{Background}

Echinoderms are well known for their outstanding capacity to regenerate parts of their body, including the central nervous system (CNS), in response to injury or autotomy $[1,2]$. The cellular and molecular events that allow echinoderms to restore the integrity of their organs and appendages with such efficiency have been a major focus in echinoderm regenerative biology. It has been established that the earliest response to injury involves extensive dedifferentiation of adult tissues, when mature

\footnotetext{
*Correspondence: vladimir.mashanov@unf.edu

${ }^{\dagger}$ Equal contributors

${ }^{1}$ University of North Florida, 1 UNF Drive, 32224 Jacksonville, FL, USA

${ }^{2}$ University of Puerto Rico, Rio Piedras, PO Box 70377, 00936-8377 San Juan, PR, USA
}

cells greatly simplify their shape and internal organization through elimination of specialized cytoplasmic structures $[1,3,4]$. Dedifferention primes the adult tissues for the subsequent morphogenetic phase, which in turn involves two distinct mechanisms to accumulate sufficient cellular material for regeneration per se - production of new cell mass through cell proliferation and rearrangement of existing cells through cell migration. The relative importance of these mechanisms remains unclear. Extensive cell division has been documented in virtually all regenerative events in echinoderms studied so far and has been investigated in great detail using thymidine analogs, such as tritiated thymidine, 5-bromo2'-deoxyuridine (BrdU), and 5-ethynyl-2'-deoxyuridine (EdU) [4-8]. On the other hand, the contribution of cell migration to regeneration has often been merely inferred 
on the basis of morphological analysis of fixed tissue samples taken at different time points after injury or autotomy $[1,5,8-10]$, but never demonstrated directly. We, therefore, do not know how crucial cell migration is for the success of the regenerative response in echinoderms. Can regeneration be still sustained if cell proliferation is abolished or significantly reduced?

One of the most interesting regenerative phenomena in echinoderms is post-traumatic neurogenesis in their injured CNS. In sea cucumbers, for example, the radial nerve cords (RNCs) fully reconnect and restore their normal cellular architecture within about 3 weeks after complete transection $[4,11]$. Each of the two RNC stumps reorganizes its lesioned surface to form a tubular outgrowth (also referred below to as 'rudiment' or 'regenerate') that invades the connective tissue matrix deposited in the wound gap. The two rudiments thus grow towards each other from the opposite sides of the wound and eventually fuse to restore the anatomical connectivity of the injured RNC. One of the most prominent cellular events that unfolds in the lesioned nervous tissue is a ten-fold increase in the number of proliferating radial glial cells (from $\sim 0.5 \%$ in uninjured animals to more than $5 \%$ during the growth phase of regeneration, as measured by BrdU incorporation). This burst of cell proliferation has therefore been considered the only major driving force in echinoderm neural regeneration [4]. However, more recent studies of cell turnover in the uninjured adult CNS suggested that cell division may not be the only cellular process implicated in neurogenesis. Some of the newborn cells generated in the normal RNC undergo extensive migration within the neuroepithelium to move away from the place of their birth and populate new locations within the nervous tissue [12]. We therefore asked if cell migration can also contribute to post-traumatic neural regeneration in echinoderms.

Here, we uncoupled the effect of proliferation from cell migration by using a pharmacological agent, aphidicolin, which inhibits DNA-polymerases of B-family and thus blocks DNA replication in the S-phase of the cell cycle. Unlike many other drugs, which often affect multiple pathways, the effect of aphidicolin is highly specific. In particular, it does not affect cell migration, RNA or protein synthesis. Aphidicolin treatment causes actively cycling cells to pause at the G1/S transition or stop DNA synthesis, if they have already entered the S-phase [13]. We demonstrated that, in spite of significant reduction in cell division, the aphidicolin-treated animals successfully formed the outgrowths at the wound surface of their RNC. Surprisingly, those regenerates were of the same absolute size and contained the same number of cells as in the control animals. We concluded that the block of cell division was compensated for by cell migration toward the growing rudiments. We then used vital cell labeling with
DiI to show that cell migration was indeed taking place in both the uninjured CNS and in post-traumatic RNC regeneration.

\section{Methods}

\section{Animal collection and maintenance}

Adult individuals of the Caribbean brown rock sea cucumber Holothuria glaberrima Selenka, 1867 (Echinodermata: Holothuroidea) were collected by hand from the shallow waters of the rocky intertidal zone of northeastern Puerto Rico (the Old San Juan area). For the duration of the experiment, the animals were kept at room temperature in indoor tanks with aerated natural sea water, which was changed weekly.

\section{Inhibition of cell division in neural regeneration}

Aphidicolin was purchased from Sigma Aldrich (A0781) and dissolved in dimethyl sulfoxide (DMSO) to a concentration of $10 \mathrm{mg} / \mathrm{mL}(0.03 \mathrm{M})$. This stock solution was stored at $-20^{\circ} \mathrm{C}$ until needed, but no longer than a month. The RNC injury was performed as described elsewhere $[4,14,15]$. Briefly, the animals were anesthetized in $0.2 \%$ chlorobutanol (Sigma 112054). The inner side of the body wall was exposed through the cloaca by pushing a glass rod against the epidermis of the "ventral" mid-body region. The RNC was cut from the coelomic side of the body wall using a sharp razor blade, and the animals were returned to the aquaria to regenerate.

To inhibit cell division, we injected aphidicolin at a dosage of $8.3 \mu \mathrm{g} / \mathrm{g}(25 \mu \mathrm{M})$ into the main body cavity. Each animal received a total of 15 injections. The first injection was done 24 hours post-injury, followed by 14 more daily injections until day 15 . The aphidicolin treatment, therefore, covered the phases of regeneration, which coincided with the formation and growth of the rudiment and involved highest levels of cell proliferation in the regenerating tissues [4]. These injection parameters were established after a series of pilot experiments (Additional file 1), in which a saturation point was reached suggesting that any further increase in drug concentration or in the number of injections will not result in stronger inhibition of cell proliferation. The first injection delivered aphidicolin alone, all subsequent injections also included $\mathrm{BrdU}(50 \mathrm{mg} / \mathrm{kg})$ to monitor the effect of aphidicolin on the S-phase DNA synthesis. Control animals received DMSO (vehicle) injections. Four animals were injected with the inhibitor and four control animals were injected with the vehicle.

On day 16 of regeneration (i.e., 24 hours after the last aphidicolin/BrdU injection), the tissue samples were fixed overnight at $4^{\circ} \mathrm{C}$ in $4 \%$ paraformaldehyde in $0.01 \mathrm{M}$ PBS ( $\mathrm{pH} 7.4)$, cryoprotected in a series of graded sucrose solutions, embedded in the OCT medium (Sakura), and cryosectioned at $10 \mu \mathrm{m}$. Prior to BrdU 
immunocytochemistry, the cryosections were postfixed for $15 \mathrm{~min}$ in formalin vapors. The slides were then rinsed in PBS, pretreated with $0.5 \%$ Triton X-100 and soaked in $2 \mathrm{~N} \mathrm{HCl}$ for $30 \mathrm{~min}$ at $37^{\circ} \mathrm{C}$. After neutralization in 0.1 $\mathrm{M}$ borate buffer, autofluorescence was quenched by incubation in $0.1 \mathrm{M}$ glycine at room temperature for 1 hour. The sections were then blocked in $2 \%$ goat serum for 1 hour and incubated overnight at $4^{\circ} \mathrm{C}$ in a mixture of a primary rat monoclonal anti-BrdU antibody (1:400, GenWay) and the echinoderm glial marker ERG1 (1:1) [16]. After extensive washes in PBS $(10 \times 10 \mathrm{~min})$, the secondary antibodies (AMCA-conjugated goat anti-rat, 1:50, Jackson ImmunoResearch Laboratories, Inc and FITC-conjugated goat anti-mouse, 1:100, BioSource) were applied for 1 hour at room temperature. Following the second round of washes $(4 \times 10 \mathrm{~min})$, the nuclei were stained with propidium iodide, and the sections were mounted in an anti-fading medium (2.5\% DABCO, $10 \%$ Mowiol 4-88 in $25 \%$ glycerol buffered with $0.2 \mathrm{M}$ Tris-HCL, $\mathrm{pH} 8.5$ ).

The immunostained sections were photographed with a Nikon Eclipse Ni microscope equipped with a DsQi2 camera using a $40 \times$ objective. Cells were counted in acquired images of every third serial section, at least seven sections per animal, using the CellCounter plugin in the Image//Fiji image analysis software [17]. The cells were counted in the regenerate per se (including the outgrowth and the zone of glial dedifferentiation) plus 100 $\mu \mathrm{m}$ of adjacent stump tissue, i.e., the region whose general histological architecture did not change after the injury (Fig. 1e, f). The size of the sampling region was measured using standard ImageJ tools.

Statistical analysis of the numerical data was performed in $\mathrm{R}$ (version 3.3.1) [18]. The raw data are available in the spreadsheet format (Additional file 2). The sample $\mathrm{R}$ code that can be used to reproduce our statistical computations, as well as the $\mathrm{R}$ output are available in Additional file 3. The two-sample Welch t-test was used to test the statistical significance of the aphidicolin treatment on a number of parameters in the regenerating RNC, including the abundance of $\mathrm{BrdU}^{+}$-cells, cell density, absolute cell number, and the length of the outgrowth. The data are represented as whisker plots in Fig. 1a-d, where the box shows the interquartile range (the values between the $25 \%$ and $75 \%$ percentiles), the thick line in the box is the median, the whiskers are drawn to the data points located within $1.5 \times$ interquartile range outside of the box.

\section{Tracing cell migration with Dil labeling}

DiI (1,1'-Dioctadecyl-3,3,3,'3'-Tetramethylindocarbocyanine Perchlorate, ThermoFisher Scientific D282) was dissolved to a final concentration of $2 \mathrm{mg} / \mathrm{mL}$ in absolute ethanol. Fine glass needles were hand-pulled from $1 \mathrm{~mm}$ diameter glass capillaries. They were dipped into the DiI ethanol solution and the ethanol was allowed to evaporate thus leaving the outer surface of the glass needle covered with a thin layer of DiI. This dye coating procedure was repeated twice for each needle.

Two different labeling strategies were used. The first one was designed to label cells at the wound margin. The RNC was injured by complete transection as described above. Immediately after making the incision, the exposed tissues on one side of the wound gap, including the radial nerve cord, were labeled by touching them with a DiIcovered glass needle. The opposite side of the wound was left unlabeled (Fig. 2a, a'). Five animals were sacrificed at each of the two time points after the surgery - on day 2 and day 25 post-injury. The tissue samples were fixed in a mixture of $2 \%$ paraformaldehyde and $0.5 \%$ glutaraldehyde in $0.01 \mathrm{M}$ PBS (pH 7.4), cryoprotected as above, and cryosectioned at 10 or $20 \mu \mathrm{m}$. Sections were collected on gelatinized slides, dried for a few minutes at room temperature and then viewed and photographed without coverslipping [19]. All sections shown in this paper are longitudinal (i.e., cut along the long axis of the radial nerve cord).

The second cell migration tracking strategy involved labeling the cells of the RNC at a distance of about $2 \mathrm{~mm}$ away from the wound margin (Fig. 3a, a') to test if those deeper cells would migrate towards the wound and contribute to regeneration. The animals were anesthetized as above. The radial nerve cord was pricked by a glass needle soaked in DiI solution. The needle was inserted from the inner (coelomic) side of the body wall and, therefore, had to pass trough the coelomic epithelium, radial water-vascular canal, and the radial hemal lacuna before reaching the radial nerve. A single transverse cut was made $2 \mathrm{~mm}$ away from the labeling site. The animals were sacrificed on days 2, 16, and 25 after labeling and surgery. At least three animals were used at each time point. The tissue samples were processed, sectioned and analyzed as above. We also included three "sham" individuals into the experimental design. The RNC of these animals was labeled by piercing with a DiI-soaked needle as above, but was not subjected to transection. These animals were analyzed on day 25 after labeling.

\section{Results}

\section{Aphidicolin reduces cell proliferation in neural} regeneration, but does not affect the size of the regenerate Our previous research indicated a significant increase in cell proliferation that accompanied the growth phase of neural regeneration in sea cucumbers $[4,11]$. It remained unclear, however, whether or not the burst in cell division was the only cellular mechanism involved in formation of the outgrowth at the wound surface of the injured RNC. In order to suppress cell division, we used aphidicolin, an inhibitor of the S-phase DNA synthesis. The treatment was designed so as to continuously inhibit cell division 

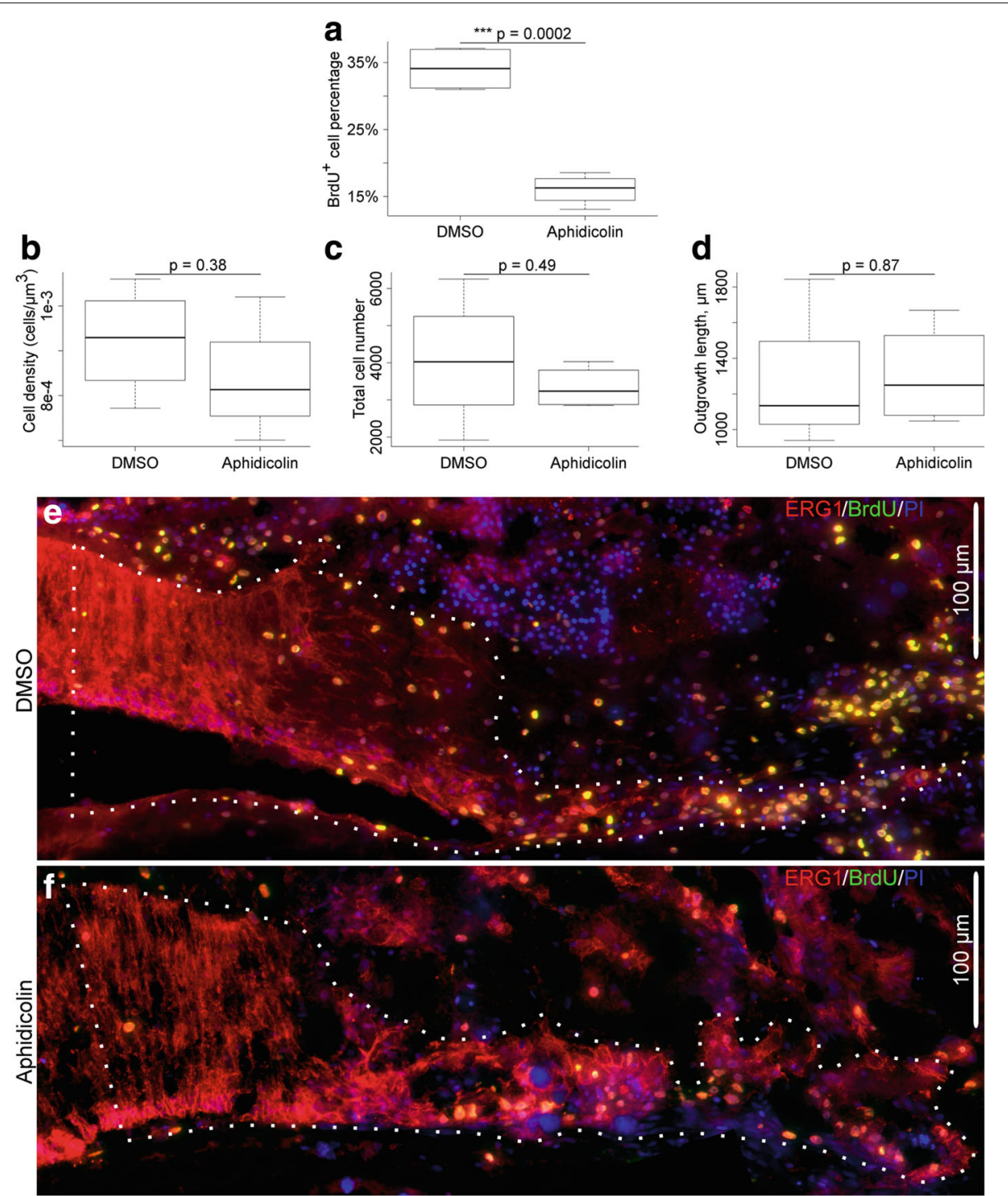

Fig. 1 Effect of aphidicolin treatment on radial nerve cord regeneration, day 16 post-injury. a The aphidicolin treatment significantly reduced (by a factor of two) the number of BrdU+ rudiment (c), nor the length of the regenerate. e and (f) show representative micrographs of the regenerating RNC in a control animal and in an animal injected with aphidicolin, respectively. The growing tip is on the right. These micrographs are merged images showing three different channels simultaneously: ERG1 (an antibody labeling echinoderm radial glia) in red, BrdU in green, and propidium iodide (PI) (nuclear stain) in blue. These channels are shown separately in Additional File 4. The dotted lines in $(\mathbf{e})$ and $(\mathbf{f})$ mark the area where cells were counted (the distal regenerate and $100 \mu \mathrm{m}$ of the proximal stump tissue - see "Methods")

from the early post-injury phase thru the late outgrowth stage. We have previously showed that cell proliferation in the regenerating RNC of $H$. glaberrima starts to increase on days 6-8 post-injury and reaches its peak on days $12-$ 14 post-injury, before returning to the background levels at later stages [4]. For 15 days after injury, the animals were daily injected with either aphidicolin dissolved in DMSO (the treatment group) or with a corresponding amount of DMSO alone (the control group). In addition, to monitor the effect of aphidicolin on the replicative DNA synthesis and thus on cell proliferation, all animals were co-injected with BrdU. As could be expected, aphidicolin treatment resulted in a highly significant 2.1 -fold decrease in the abundance of BrdU-positive cells in the regenerating RNC $(16.05 \pm 1.14 \%$ vs $34.06 \pm 1.66 \%$, mean \pm standard error, in aphidicolin-treated and control DMSO-treated animals, respectively) (Fig. 1a, e, f, Additional file 4).

Surprisingly, in spite of the significant inhibition of cell division, the regenerating RNC in the aphidicolintreated individuals had the same total length, absolute cell number and cell density as in the control individuals (Fig. 1b-f). Besides, the growing tips of the regenerating 

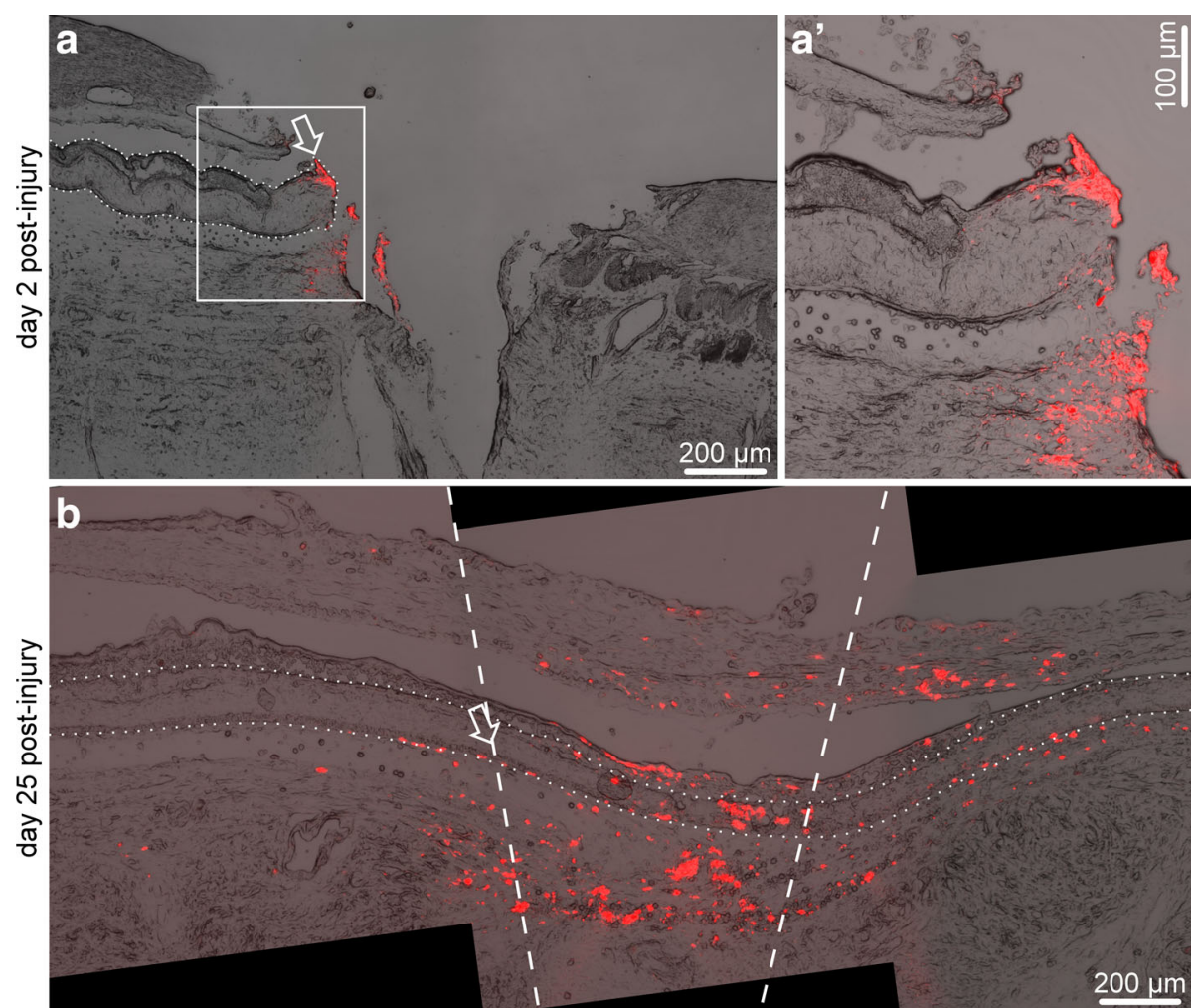

Fig. 2 Unilateral Dil labeling of the injured radial nerve cord, longitudinal sections. Immediately after bisection, the dye was applied to one side of the wound, while the opposite side was left unlabeled. The arrow shows the site of the original dye application. $\mathbf{a}$ On day 2 after the surgery, the dye remained on one side of the wound. (a') Higher magnification of the boxed area in (a). b By day 25 after surgery, the wound gap is bridged and the two stumps of the radial nerve cord have reconnected across the wound gap. The dashed lines show the original wound margins. Note extensive migration of labeled cells into the new tissue bridging the wound gap. The dotted line marks the outline of the radial nerve cord

RNCs in both the treatment and control groups showed the same overall tissue architecture (compare Fig. 1e and f) and were composed, as previously described [4, 11], of a flattened epithelium made up of dedifferentiated radial glial cells.

How does the regenerating tip of the RNC maintain its size, geometry and cell number when cell division is inhibited? We hypothesized that in addition to cell proliferation in situ, there is an additional cell source contributing to regeneration - migrating cells from the more distant regions of the nervous tissue.

\section{Cell migration contributes to radial nerve cord regeneration and also happens in the uninured CNS}

In order to study cell migration in vivo in the regenerating RNC, we employed vital staining with the lipophilic dye DiI. Two labeling experiments were carried out - one to trace the cells at the wound margin (Fig. 2), and the other to label deeper cells located at a distance of about $2 \mathrm{~mm}$ away from the plane of injury (Fig. 3a-c).

Two days after the surgery and dye application to the cells at the wound margin, five animals were sacrificed and examined to assess the technical success of the first labeling strategy. In all five individuals, DiI-marked cells were seen at one side of the wound only, while the opposite side remained clear (Fig. 2a, a'). By day 25 after injury, the regenerating RNC completely re-connected across the injury gap, and the labeled cells of the wound margin not only have migrated to populate the newly created segment of the RNC, but also moved all the way across the wound gap into the RNC stump at the opposite side of the wound, up to $\sim 1,300 \mu \mathrm{m}$ from the site of original labeling (Fig. 2b), as measured in cryosections.

The second labeling strategy involved delivery of the dye to deeper cells of the RNC stump, located about $2 \mathrm{~mm}$ away from the wound margin (Fig. 3a, a'). Note however that due to fixation and tissue processing, the absolute distances are shorter when measured in cryosections. On day 16 after injury and dye application, the DiI-labeled cells are seen throughout the growing tip of the regenerating RNC (Fig. 3b, b'). In the fully regenerated RNC on day 25 post-injury, many DiI-labeled cells are scattered between the site of dye application and the injury plane and also in the newly created segment of the RNC spanning the injury gap (Fig. 3c), suggesting that deep cells are capable 

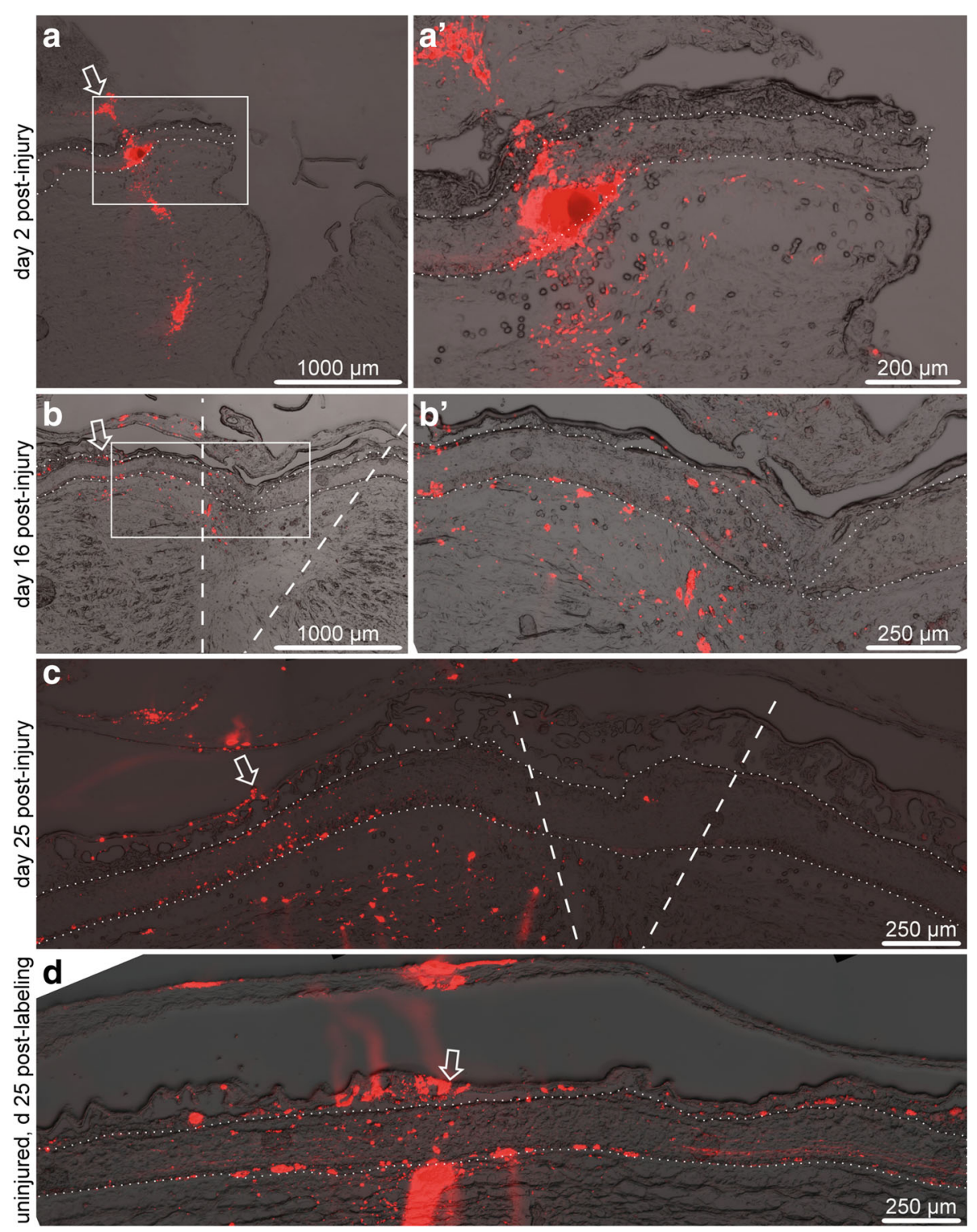

Fig. 3 Dil labeling at a distance of $2 \mathrm{~mm}$ from the cut on days $2\left(\mathbf{a}, \mathrm{a}^{\prime}\right), 16\left(\mathbf{b}, \mathrm{b}^{\prime}\right)$, and $25(\mathbf{c})$ after labeling and injury. The site of dye application is indicated by an arrow. The radial nerve cord is outlined by dotted lines. Dashed lines show the original wound magins. ( $\left.a^{\prime}\right)$ and ( $\left.b^{\prime}\right)$ are higher magnification views of the boxed regions in $\mathbf{a}$ and $\mathbf{b}$, respectively. $\mathbf{d}$ Uninjured radial nerve cord 25 days after labeling

of migrating towards the wound and contributing to RNC regeneration.

We have previously suggested that cell migration also takes place in the uninjured sea cucumber CNS under normal physiological conditions. That conclusion was based on observations of changes in distribution of BrdUpositive cells in animals left for long chase periods [12]. Here, we confirmed those earlier observations with vital dye labeling. To this end, we pricked the uninjured midventral RNC at the mid-body level with a DiI-soaked glass needle and kept the animals alive for 25 days. After fixation and cryosectioning, the DiI labeling cells were seen scattered over a distance of over $1,000 \mu \mathrm{m}$ in both directions along the anterior-posterior axis from the site of dye application (Fig. $3 \mathrm{~d}$ ), suggesting that some cell migration occurs even in the uninjured RNC.

\section{Discussion}

Our results indicate that neural regeneration in echinoderms is a robust and regulative developmental process. In our experiments, a more than two-fold decrease in cell proliferation did not result in a delayed regeneration or in a smaller or deformed RNC outgrowth. On the contrary, the regenerating RNC in aphidicolin-treated 
animals could still amass the same number of cells and grow to the same absolute size as in the control animals. The only explanation that we can propose is that the deficiency in cell division in the rudiment was compensated for by recruitment of cells through migration.

To directly demonstrate that cell migration does in fact take place, we used the lipophilic vital dye DiI. DiI is irreversibly taken up by cell membranes, without any toxic effect, and, importantly, the labeling does not diffuse from one cell to another [19]. Two distinct types of cell migration have been implicated in echinoderms tissue regeneration: epithelial invasion, or collective migration of epithelial cells joined by intercellular junctions $[4,9,11]$, and migration of individual mesenchymal cells $[2,8,10]$. So far, it has been proposed that the echinoderm RNC regenerates by reorganizing the cells at the wound site to form a thin tubular outgrowth that later develops into a new RNC segment. The outgrowth is composed of glial cells, which greatly simplify their organization and undergo extensive cell division, but nevertheless maintain intercellular junctions. Epithelial invasion has therefore been proposed as the primary mode of RNC extension across the wound gap. The present study suggest that it might not be the only mechanism. The fact that some of the cells that take up DiI at the wound surface immediately after RNC transection (the first labeling strategy, see Results) are found at later stages of regeneration not only in the newly created segment of the RNC that bridges the injury gap, but also within the "old" former stump RNC region on the opposite side of the wound, suggest that some of the wound surface cells are capable of extensive migration as individual cells. Likewise, migration of "deep" RNC cells from a distance of 2 $\mathrm{mm}$ (which equals about 400 neuronal or glial cell body diameters) from the plane of the transection (the second labeling strategy, see Results) towards and beyond the plane of injury can only be accomplished by individual cells that are not anchored to their neighbors by intercellular junctions. Migration of individual cells through the neuroepithelium to new locations, therefore, contributes to redistiribution of cells within the regenerating CNS.

The present study did not clearly establish the identity of the migrating cells. Nevertheless, the available evidence allows us to rule out some of the potential candidates. First, the migrating cells are not mature neurons. For reasons that are still unknown to us, our DiI labeling procedure does not usually yield "typical" outcome of whole-cell neuronal labeling seen in vertebrates. Only on very rare occasions do we see labeled axonal tracts. Second, due to technical limitations of our surgical procedure we could not avoid labeling some of the mesenchymal cells in the connective tissue regions surrounding the RNC. Even though these cells are capable of extensive migration, our previous studies showed that they make no significant contribution to RNC regeneration [11]. The exact nature of the migratory cells in post-traumatic neurogenesis in echinoderms remains an important question to be resolved by future studies.

In spite of the chronic exposure to aphidicolin, the cell division in the $\mathrm{RNC}$ regenerate, although decreased more than two-fold, did not stop completely. We do not think that the inability of our aphidicolin treatment to completely abolish cell division was due to insufficient concentration of the drug or the duration of the procedure. First, the dosage that we used $(25 \mu \mathrm{M})$ was similar or higher than the range of concentrations used in similar experiments with echinoderm and vertebrate embryos, larvae, organ and cell cultures $(0.6-30 \mu \mathrm{M})$, which achieved complete or near-complete cell division inhibition [2024]. Second, our preliminary experiments showed that treatments at lower doses and smaller number of injection caused a similar two-fold decrease in mitotic activity in RNC regeneration. The increase in dosage and in the number of injection, however, resulted in much smaller variation in response to the treatment between individuals. Third, even at a much higher concentration $(150 \mu \mathrm{M})$ aphidicolin did not completely stop cell division in Xenopus embryos. The authors suggested that this observation was due to some residual DNA synthesis, which allowed the cells to complete the S-phase and go into the mitotic cycle [25]. Depending on the specific properties of an organism, cell division inhibitors are known to vary in extent of their effect on cell proliferation. For example, one of the factors that prevents the general use of aphidicolin as an anticancer drug in human patients is its very rapid clearance from the human plasma [13]. The partial resistance of the regenerating echinoderm nervous tissue to aphidicolin treatment may provide some interesting insights into DNA replication biology and physiology of echinoderms.

The present study, therefore, directly demonstrates that migration of individual cells plays an important role in post-traumatic neurogenesis in echinoderms and presumably can rescue regenerative response if cell proliferation is suppressed. This report adds to the growing realization that migration of individual cells is a significant, although traditionally underappreciated and often improperly studied, factor contributing to post-embryonic developmental phenomena, such as post-traumatic regeneration and normal cell replenishment, in a wide range of multicellular animals [26].

\section{Conclusions}

- Chronic aphidicolin treatment resulted in a significant decrease in cell proliferation in the regenerating RNC of the sea cucumber $H$. glaberrima

- The RNC in the aphidicolin-treated animals, however, continued to regenerate and did not differ 
from the vehicle-treated controls in size, internal structure, or cell number.

- We speculate that the inhibition of cell division was compensated for by cell migration from more distant regions of the neuroepithelium located as far as $2 \mathrm{~mm}$ from the site of the original injury and outgrowth formation.

\section{Additional files}

Additional file 1: Data from two pilot experiments that were carried out to determine the dosage of aphidicolin and injection schedule. (PDF 447 kb)

Additional file 2: Spreadsheet (.xlsx) file containing the raw measurements of $\mathrm{BrdU}^{+}$-cell abundance, total cell number and cell density in the radial nerve cord regenerate, as well as the outgrowth length. (XLSX $11.3 \mathrm{~kb}$ )

Additional file 3: $R$ code used to perform the statistical computations and generate the box and whisker plots. (R $3.11 \mathrm{~kb}$ )

Additional file 4: The same micrographs as in Fig. 1E, F shown in three separate fluorescent channels $(A)-\left(A^{\prime \prime}\right)$ Regenerating RNC in a control animal (corresponds to Fig. 1E). (B) - ( $\left.B^{\prime \prime}\right)$ Regenerating RNC in an aphidicolin-treated animal (corresponds to Fig. 1F). The top row shows ERG1 antibody staining; the second row shows BrdU immunocytochemistry; and the bottom row shows nuclear staining with propidium iodide (PI). The dotted lines mark the ares of the RNC where the cells were counted (regenerate $+100 \mu \mathrm{m}$ of the proximal stump tissue). (JPG $2846.72 \mathrm{~kb}$ )

\section{Abbreviations}

BrdU: 5-bromo-2'-deoxyuridine; Dil: 1,1'-Dioctadecyl-3,3,3',3'-Tetramethylindocar bocyanine Perchlorate; DMSO: dimethyl sulfoxide; RNC: radial nerve cord

\section{Acknowledgments}

Not applicable.

\section{Funding}

The study was supported by grants from the NIH (1SC1GM084770-01, 1R03NS065275-01) and the NSF (IOS-0842870, IOS-1252679), as well as by the University of Puerto Rico.

\section{Availability of data and materials}

All data are reported in the article and contained in additional files submitted along with the manuscript.

\section{Authors' contributions}

VSM, ORZ and JEGA conceived the study and interpreted the results. ORZ and VSM carried out experimental procedures and analyzed the data. VSM drafted the manuscript. JEGA, ORZ, and VSM finalized the manuscript. All authors read and approved the final manuscript.

\section{Competing interests}

The authors declare that they have no competing interests.

\section{Ethics approval and consent to participate}

No human subjects were involved in the study. All experiments with sea cucumbers were carried out in compliance with the NSF and NIH guidelines and with the approval of the University of Puerto Rico. The sea cucumber Holothuria glaberrima is not a regulated or endangered species.

Received: 5 August 2016 Accepted: 7 February 2017

Published online: 23 February 2017

\section{References}

1. Candia Carnevali M. Regeneration in echinoderms: repair, regrowth, cloning. Invertebr Survivial J. 2006;3:64-76.
2. Hernroth B, Farahani F, Brunborg G, Dupont S, Dejmek A, Nilsson Sköld H. Possibility of mixed progenitor cells in sea star arm regeneration. J Exp Zool B Mol Dev Evol. 2010;314B(6):457-68. doi:10.1002/jez.b.21352.

3. García-Arrarás JE, Dolmatov IY. Echinoderms: potential model systems for studies on muscle regeneration. Curr Pharm Des. 2010;16(8):942-55.

4. Mashanov VS, Zueva OR, García-Arrarás JE. Radial glial cells play a key role in echinoderm neural regeneration. BMC Biol. 2013;11(1):49.

5. Leibson NL. Regeneration of digestive tube in holothurians Stichopus japonicus and Eupentacta fraudatrix,. Monogr Dev Biol. 1992;23:51-61.

6. Candia Carnevali MD, Bonasoro F, Patruno M, Thorndyke MC. Cellular and molecular mechanisms of arm regeneration in crinoid echinoderms: the potential of arm explants. Dev Genes Evol. 1998;208(8):421-30.

7. García-Arrarás JE, Estrada-Rodgers L, Santiago R, Torres II, Díaz-Miranda L, Torres-Avillán I. Cellular mechanisms of intestine regeneration in the sea cucumber, Holothuria glaberrima Selenka (Holothuroidea: Echinodermata). J Exp Zool. 1998;281(4):288-304.

8. Czarkwiani A, Ferrario C, Dylus DV, Sugni M, Oliveri P. Skeletal regeneration in the brittle star amphiura filiformis. Front Zool. 2016;13:18. doi:10.1186/s12983-016-0149-X.

9. Mashanov VS, Dolmatov IY, Heinzeller T. Transdifferentiation in holothurian gut regeneration. Biol Bull. 2005;209(3):184-93.

10. García-Arrarás JE, Valentín-Tirado G, Flores JE, Rosa RJ, Rivera-Cruz A San Miguel-Ruiz JE, Tossas K. Cell dedifferentiation and epithelial to mesenchymal transitions during intestinal regeneration in H. glaberrima,. BMC Dev Biol. 2011;11:61. doi:10.1186/1471-213X-11-61.

11. Mashanov VS, Zueva OR, Heinzeller T. Regeneration of the radial nerve cord in a holothurian: a promising new model system for studying post-traumatic recovery in the adult nervous system. Tissue Cell. 2008;40(5):351-72. doi:10.1016/j.tice.2008.03.004.

12. Mashanov VS, Zueva OR, García-Arrarás JE. Heterogeneous generation of new cells in the adult echinoderm nervous system. Front Neuroanat. 2015;9:123. doi:10.3389/fnana.2015.00123.

13. Baranovskiy AG, Babayeva ND, Suwa Y, Gu J, Pavlov Yl, Tahirov TH. Structural basis for inhibition of dna replication by aphidicolin. Nucleic Acids Res. 2014;42(22):14013-21. doi:10.1093/nar/gku1209.

14. Mashanov VS, Zueva OR, García-Arrarás JE. Transcriptomic changes during regeneration of the central nervous system in an echinoderm. BMC Genomics. 2014;15:357. doi:10.1186/1471-2164-15-357.

15. Mashanov VS, Zueva OR, García-Arrarás JE. Myc regulates programmed cell death and radial glia dedifferentiation after neural injury in an echinoderm. BMC Dev Biol. 2015;15(1):24. doi:10.1186/s12861-015-0071-Z.

16. Mashanov VS, Zueva OR, Garcia-Arraras JE. Organization of glial cells in the adult sea cucumber central nervous system. Glia. 2010;58(13): 1581-93. doi:10.1002/glia.21031

17. Schindelin J, Arganda-Carreras I, Frise E, Kaynig V, Longair M, Pietzsch T, Preibisch S, Rueden C, Saalfeld S, Schmid B, Tinevez JY, White DJ, Hartenstein V, Eliceiri K, Tomancak P, Cardona A. Fiji: an open-source platform for biological-image analysis. Nat Methods. 2012;9(7):676-82. doi:10.1038/nmeth.2019.

18. R Core Team. R: A Language and Environment for Statistical Computing. Vienna, Austria: R Foundation for Statistical Computing; 2015. R Foundation for Statistical Computing. http://www.R-project.org/.

19. von Bartheld CS, Cunningham DE, Rubel EW. Neuronal tracing with dii: decalcification, cryosectioning, and photoconversion for light and electron microscopic analysis. J Histochem Cytochem. 1990;38(5):725-33.

20. Fishman VS, Shnayder TA, Orishchenko KE, Bader M, Alenina N, Serov OL. Cell divisions are not essential for the direct conversion of fibroblasts into neuronal cells. Cell Cycle. 2015;14(8):1188-96. doi:10.1080/15384101.2015.1012875.

21. Geling A, Itoh M, Tallafuss A, Chapouton P, Tannhäuser B, Kuwada JY, Chitnis $A B$, Bally-Cuif L. bHLH transcription factor her 5 links patterning to regional inhibition of neurogenesis at the midbrain-hindbrain boundary. Development. 2003;130(8):1591-604.

22. Krupke OA, Burke RD. Eph-ephrin signaling and focal adhesion kinase regulate actomyosin-dependent apical constriction of ciliary band cells. Development. 2014;141(5):1075-84. doi:10.1242/dev.100123.

23. Smith MS, Collins S, Raff RA. Morphogenetic mechanisms of coelom formation in the direct-developing sea urchin heliocidaris erythrogramma. Dev Genes Evol. 2009;219(1):21-9. doi:10.1007/s00427-008-0262-8. 
24. Shang J, Cafaro J, Nehmer R, Stone J. Supporting cell division is not required for regeneration of auditory hair cells after ototoxic injury in vitro. J Assoc Res Otolaryngol. 2010;11(2):203-22. doi:10.1007/s10162-009-0206-7.

25. Harris WA, Hartenstein V. Neuronal determination without cell division in xenopus embryos. Neuron. 1991;6(4):499-515.

26. Zattara EE, Turlington KW, Bely AE. Long-term time-lapse live imaging reveals extensive cell migration during annelid regeneration. BMC Dev Biol. 2016;16:6. doi:10.1186/s12861-016-0104-2.

Submit your next manuscript to BioMed Central and we will help you at every step:

- We accept pre-submission inquiries

- Our selector tool helps you to find the most relevant journal

- We provide round the clock customer support

- Convenient online submission

- Thorough peer review

- Inclusion in PubMed and all major indexing services

- Maximum visibility for your research

Submit your manuscript at www.biomedcentral.com/submit
) Biomed Central 